Original scientific paper

\title{
PERFORMANCE DIFFERENCES OF ELECTRICAL SUBMERSIBLE PUMP UNDER VARIABLE SPEED SCHEMES
}

\author{
Wang, H. L. ${ }^{*}$; Hu, Q. X. ${ }^{* *}$; Yang, Y.** \& Wang, C.***\# \\ ${ }^{*}$ School of Aerospace and Mechanical Engineering/Flight College, Changzhou Institute of Technology, \\ Changzhou, 213032, China \\ ** National Research Center of Pumps, Jiangsu University, Zhenjiang, 212013, China \\ ${ }^{* * *}$ College of Hydraulic Science and Engineering, Yangzhou University, Yangzhou, 225009, China \\ E-Mail: wangchuan198710@126.com ( ${ }^{\#}$ Corresponding author)
}

\begin{abstract}
Frequent shifts of electrical submersible pump (ESP) often lead to system vibration and noise, thereby reducing its operational stability. Three different speed change schemes were investigated to improve the stability of its variable speed process. The operational stability of ESP under different variable speed schemes was analysed. The transient numerical calculations of different variable speed schemes were conducted on ANSYS CFX to obtain the head fluctuation law. The stability of ESP with different head fluctuation laws was analysed, and the accuracy of numerical calculation was verified through tests. Result show that a high similarity is found between the head change curves and speed change curves with all methods. The head stability in uniform acceleration variable speed process and quadratic acceleration process with negative quadratic coefficient is high. The extreme value of head variation rate with the two variable speed processes is small, which is convenient for the stability of the variable speed operation of ESP. The research results serve as guide for improving the stability of the variable speed process of ESP.
\end{abstract}

(Received in October 2020, accepted in January 2021. This paper was with the authors 2 months for 1 revision.)

Key Words: Electrical Submersible Pump, Variable Speed Regulation, Transient Calculation, Numerical Simulation

\section{INTRODUCTION}

As key equipment to provide high head liquids, electrical submersible pumps (ESPs) can meet the requirements of different flow conditions. Therefore, they have been widely used in offshore oil exploitation [1,2]. Taking China as an example, the oil extraction of ESPs accounts for $90 \%$ of oil production in offshore oil fields [3,4]. An ESP needs to be placed deep into the bottom of the oilfield when in use $[5,6]$. The geology of an oilfield reservoir has strong uncertainty. This condition requires the speed adjustment of ESP based on the oilfield's real-time oil output to make the ESP's operating conditions match the oilfield conditions $[7,8]$. In actual production, the rotational speed of energy supply motor of the ESP is adjusted with a frequency converter $[9,10]$. Few studies have been conducted on the rotational speed adjustment methods of the inverter within the ESP system. However, the instantaneous characteristics of internal flow field of the ESP are gradually superimposed because its installation structure is multistage series arrangement $[11,12]$. This condition indicates that the effect of different speed adjustment methods on the transient characteristics of the flow field within the ESP cannot be ignored. The transient characteristics of the flow field inside the ESP caused by variable speed adjustment process may greatly threaten the operational stability and safety of the ESP [13, 14]. Therefore, investigating the transient changes in flow field within the ESP based on different speed regulation schemes and selecting the optimal speed regulation scheme to improve the operational stability of the ESP have become important topics in the research of ESP.

In the available literature, researchers have summarized the performance conversion and internal flow field difference patterns of pumps at different rotational speeds [15, 16]. However, studies on the effect of ESP's speed variation mode on its performance and internal flow field 
have not been reported. This study explores the changes in external characteristics and internal flow field of ESP when the rotational speed changes. The head changes in ESP with different rotational speed changes are investigated through unsteady numerical calculation to provide a certain theoretical basis for the variable speed operation of ESP.

\section{STATE OF THE ART}

Rotational speed is an important parameter of pumps. Many experts and scholars have studied speed adjustment method to improve the performance and operational stability of pumps. In medical heart pumps, conventional continuous-flow left ventricular assist devices (LVADs) reduce the pulsatility of pericardial vessels, thereby leading to side effects, such as bleeding and thrombotic events. Hydren et al. [17] found that real-time adjustment of pump speed in the LVAD system through sophisticated testing of a patient's cardiac parameters can greatly improve the performance of the LVAD system and suppress its side effects on a patient's vascular system. Schmidt et al. [18] observed that the speed of a conventional heart pump is unregulated during a patient's physical exercise. Thus, the cardiac output and exercise capacity of a patient remain significantly limited. Therefore, the team developed an automated pump speed control method based on fill pressure values that matches the pump speed to increase venous return for improving patients' exercise capacity and quality of life. Liao et al. [19] investigated the effect of blood pump speed changes on the risk of intravenous thrombosis. The real-time regulation of rotational speed improves the pulsation rate of intravenous blood, thereby minimizing blood stasis.

In industrial applications, Ge et al. [20] achieved efficiency improvement of hydraulic excavators on the basis of the speed regulation of hydraulic transfer pumps, thereby reducing noise during excavator use to a certain extent and reducing pollutant emissions. Page et al. [21] managed the fluid pressure in the entire water supply line on the basis of the regulation of pump speed. This method reduces the number of management controllers in the water supply system and optimizes the control system on the basis of hydraulic theory based on pump speed adjustment. Nag and Lee [22] maximized the efficiency of the system by using a real-time variable speed approach as much as the maximum power allowed. The doubly-fed induction machine (DFIM) method used in this work is effective in increasing the system performance at some speeds and in improving the stability of the system during fixed-speed operation. Morabito et al. [23] designed a hydraulic turbine for the variable speed regulation of pump. This variable speed system can avoid the instability of full load operation, greatly improve the operating life of the pump when it is used as a hydraulic turbine, and enhance the overall efficiency and operating stability of the system. The method based on variable speed regulation to achieve pump and pump system performance improvement is highly feasible.

To improve the reliability of variable speed regulation, Bai et al. [24] conducted numerical calculations and experimental validation of a high flow rate and high power rescue pump at different speeds. The results show the inlet and outlet angles of the impeller vanes match well with the flow angle of the liquid. The pressure pulsation in the pump at different rotational speeds is analysed. The pressure pulsation coefficient in the pump increases obviously with the increase in rotational speed and is mostly a low frequency signal. The pressure pulsation is the cause of the instability of pump operation. Al-Obaidi [25] studied the effect of centrifugal pump speed on its performance and cavitation characteristics through vibration analysis. He found that as the pump speed increases, the cavitation performance of the pump itself decreases, the vibration intensity of its pump body increases, and the frequency of vibration changes significantly. Li et al. [26] conducted numerical calculations and experimental analysis on the performance of an automotive water pump at different rotational speeds. Its head and maximum efficiency point increase with the increase in rotational speed. However, its impeller is a semi- 
open structure, and the intensity of leakage flow at the top of the impeller increases with the increase in rotational speed. The vortex structure causes large hydraulic losses in the pump impeller and leads to a significant decrease in the anticavitation performance of the automotive water pump. Wang et al. [27] conducted experimental measurements on the performance and pressure pulsation characteristics of double suction pumps at different speeds. They confirmed the correspondence between the main frequency and speed in pressure pulsation, which is beneficial to the hydraulic design of double suction pumps and to obtain a stable hydraulic model. Yang et al. [28] conducted a steady-state analysis on the performance of ESP at different speeds. The head of ESP at different speeds basically satisfies the similar law without considering the volume loss. However, its shaft power deviates from the prediction of the similar law to some extent. This condition is because the disk friction loss at different speeds does not satisfy the similar law. This finding serves as reference for the subsequent prediction of ESP performance correction at different speeds. Han and Tan [29] found that for high specific speed centrifugal pumps, the pump head conforms to a similar law with the increase in speed. The pump efficiency shifts toward large flow rates. However, the intensity of leakage flow at the top of the lobe and the oscillation frequency of the leakage vortex increase with the increase in speed. The percentage of space in the flow path increases. This work provides a concrete work on the effect of speed increase on pump leakage flow, but does not quantify the extent to which the increase in leakage vortex intensity affects the pump stability.

The above studies all focus on the performance differences and operational stability of the pump at different speeds. The numerical calculations are steady calculations at different speeds. However, the performance changes and stability of the pump during the variable speed process are not mentioned. The pump speed variation process has extremely strong transient characteristics and is closely related to the pump operating stability. This study explores the effect of different speed variation schemes on pump performance and stability by establishing a numerical model of ESP. The performance and stability changes are investigated during the speed variation of ESP to find the most favourable speed variation scheme for the stable operation of ESP.

The remainder of this paper is arranged as follows: Section 3 discusses the modelling for pump parameter optimization and mesh generation and the experimental validation of numerical simulation. Section 4 obtains the performance of ESP with different speed variation schemes and analyses the advantages and disadvantages between the different results. Section 5 summarizes the study and provides the conclusions.

\section{METHODOLOGY}

\subsection{Geometric model}

Although hundreds or thousands of stages may be found in the actual application of an ESP unit, the geometric structure between the stages is exactly the same. Three-stage modelling of a KF480 type ESP was conducted on the professional 3D modelling software NX UG in this study. The impeller, diffuser, and chamber subdomains were included in each stage of the computational domain. The import and export sections were arranged before the first stage and after the last stage. The model of the inlet and outlet sections was simplified as circular straight pipes where their diameter is equal to the pipeline diameter within the ESP system. The lengths of inlet and outlet sections are greater than five times of the impeller inlet diameter to ensure that the liquid medium has sufficient space for flow evolution that can distinctly enhance the accuracy of numerical simulations. 


\subsection{Numerical simulation}

Ansys TurboGrid was used for the structural meshing of the impeller and diffuser to obtain a high-quality hexahedral mesh. The structural meshing of the pump chamber, inlet, and outlet sections was conducted on Ansys ICEM. High-quality boundary layer meshes were generated at all wall locations to ensure the accuracy of numerical calculations. Fig. 1 shows the mesh details of the impeller and diffuser. The quantity and quality of the mesh directly affect the accuracy and time duration of numerical calculations. The method of controlling the mesh global maximum size was adopted to verify the grid number independence in this article. The mesh quality of each group used for independence verification is greater than 0.4. The verification results of different mesh size schemes are shown in Table I. When the maximum control size of the mesh is less than or equal to $1.1 \mathrm{~mm}$, the fluctuation of ESP's performance prediction result is than $0.3 \%$. Thus, $1.1 \mathrm{~mm}$ was chosen as the global maximum control size of the mesh.

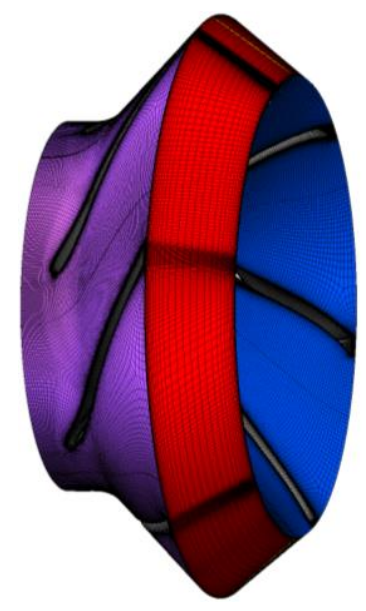

a) Impeller

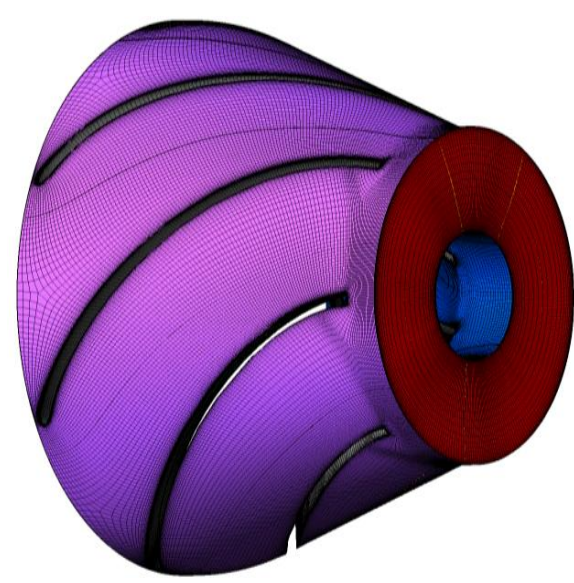

b) Diffuser

Figure 1: Mesh details of impeller and diffuser.

Table I: Mesh independence analysis.

\begin{tabular}{|c|c|c|c|c|}
\hline \multirow{2}{*}{$\begin{array}{c}\text { Maximum } \\
\text { size }(\mathrm{mm})\end{array}$} & \multicolumn{2}{|c|}{ Number of meshes } & \multicolumn{2}{c|}{ Predicted results } \\
\cline { 2 - 5 } & Impeller & Diffuser & Head $(\mathrm{m})$ & Efficiency $(\%)$ \\
\hline 1.5 & 50,030 & 86,428 & 44.04 & 76.21 \\
\hline 1.3 & 190,546 & 294,568 & 44.09 & 76.35 \\
\hline 1.1 & 318,645 & 504,960 & 44.12 & 76.48 \\
\hline 0.9 & 730,895 & $1,297,380$ & 44.11 & 76.53 \\
\hline
\end{tabular}

The numerical calculations in this article were all conducted on Ansys-CFX 17.0. Constant numerical calculations were performed. Their results were compared with the experimental performance of ESP to verify the accuracy of numerical simulation. The convergence accuracy of constant calculations was set to $10^{-4}$. This condition can provide stable initial flow field conditions for subsequent nonconstant numerical calculations and shorten the time of nonconstant numerical calculations. The no-slip boundary was adopted. Different subdomains were connected by interface, where "general connection: no frame change" was chosen when no relative motion was found between adjacent subdomains, and "general connection: pitch change" was chosen when relative motion was found between adjacent subdomains. The mass flow rate outlet with a total pressure inlet was used for easy convergence. The initial pressure within domains was set to standard atmospheric pressure. The location and type of the computational domain model and boundary conditions are shown in Fig. 2. 
The subsequent variable speed transient numerical calculations were set on the basis of the constant numerical calculation settings. The calculation property was changed to transient, and the total time and timestep of the calculation were defined. The capturing of transient flow characteristics and the computer configuration need to be considered when we set the timestep. Capturing the transient characteristics of the flow field with extremely large timestep is unconducive, whereas it requires high computer performance with extremely small timestep. In the constant numerical calculations, the rotation of the impeller domain is the rotation of the coordinate system. The intersection between the impeller domain and other stationary domains is set as "frozen rotor". However, in the transient numerical calculations, the phase angle between the impeller and the stationary domain is changing all the time. Thus, the intersection between them needs to be set as "transient rotor stator".

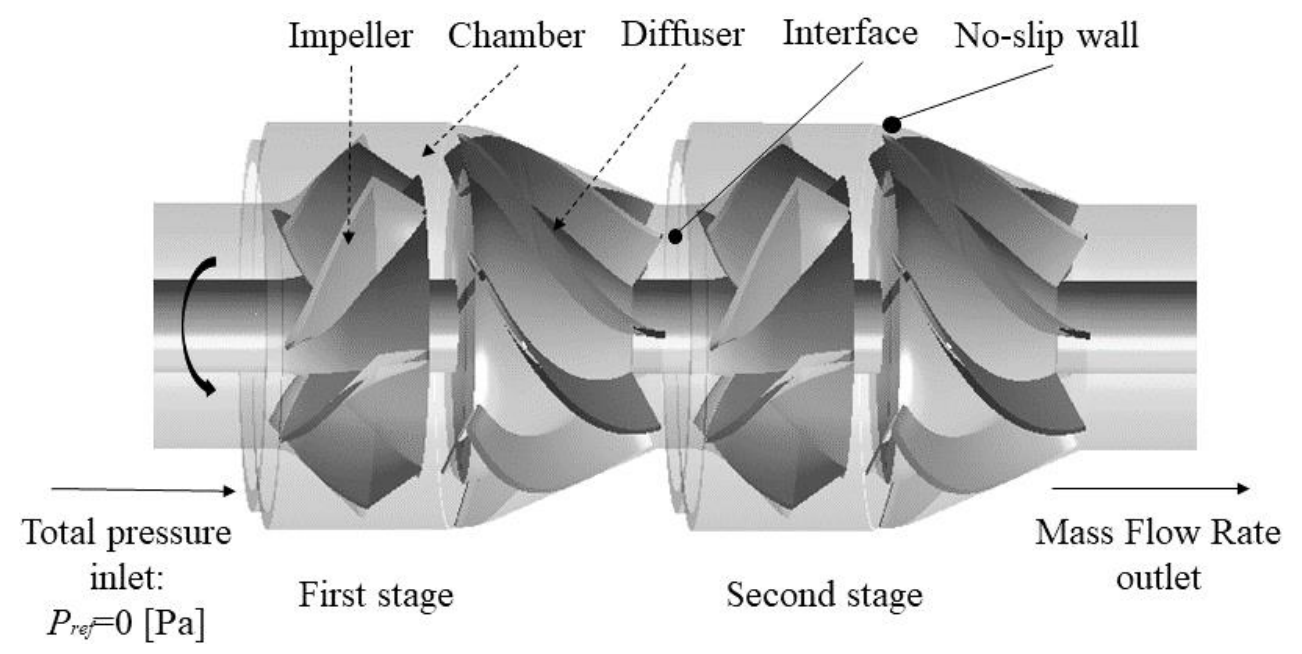

Figure 2: Calculation domain and boundary conditions.

The standard $k-\varepsilon$ model is the widely used turbulence model in engineering applications $[30,31]$ because it is stable and relatively accurate. However, the $\varepsilon$ equations in the standard $k-\varepsilon$ model include a term that cannot be calculated on the wall [32]. Thus, the standard $k-\varepsilon$ model with standard wall function is used to complete the numerical calculation. The equations can be expressed as follows:

- For turbulent kinetic energy $k$ :

$$
\frac{\partial(\rho k)}{\partial t}+\frac{\partial\left(\rho k u_{i}\right)}{\partial t}=\frac{\partial}{\partial x_{j}}\left[\left(\mu+\frac{\mu_{i}}{\sigma_{k}}\right) \frac{\partial k}{\partial x_{j}}\right]+G_{k}+G_{b}-Y_{M}-\rho \varepsilon+S_{k}
$$

- For dissipation $\varepsilon$ :

$$
\frac{\partial(\rho \varepsilon)}{\partial t}+\frac{\partial\left(\rho \varepsilon u_{i}\right)}{\partial x_{i}}=\frac{\partial}{\partial x_{j}}\left[\left(\mu+\frac{\mu_{i}}{\sigma_{\varepsilon}}\right) \frac{\partial \varepsilon}{\partial x_{j}}\right]+\frac{C_{1 \varepsilon} \varepsilon}{k}\left(G_{k}+G_{3 \varepsilon} G_{b}\right)-\rho C_{2 \varepsilon} \frac{\varepsilon^{2}}{k}+S_{\varepsilon}
$$

\subsection{Experimental validation of numerical simulation}

The performance test of KF480 type ESP was conducted to verify the accuracy of numerical calculations. The rated speed of ESP during the test is $6000 \mathrm{r} / \mathrm{min}$. Fig. 3 shows the test system installation. The pipeline turbine flowmeter in the test system can perform instantaneous measurement of flow with an accuracy of 0.5 grade and a nominal pressure of $1.6 \mathrm{MPa}$. The three-phase power meter can complete the power measurement with an electric dynamometer. Its effective power measurement ranges from $1 \mathrm{~kW}$ to $24 \mathrm{~kW}$ with 1.5 grade measurement accuracy. A precision pressure sensor with an accuracy class of 0.4 is arranged at the outlet to 
ensure the accuracy of pressure measurement. Fig. 4 shows the solid model of the impeller and diffuser used in the test. The impeller and diffuser are made of high-strength plastic by rapid moulding technology. This condition can meet the strength requirements of the test parts and shorten the preparation time of the test.

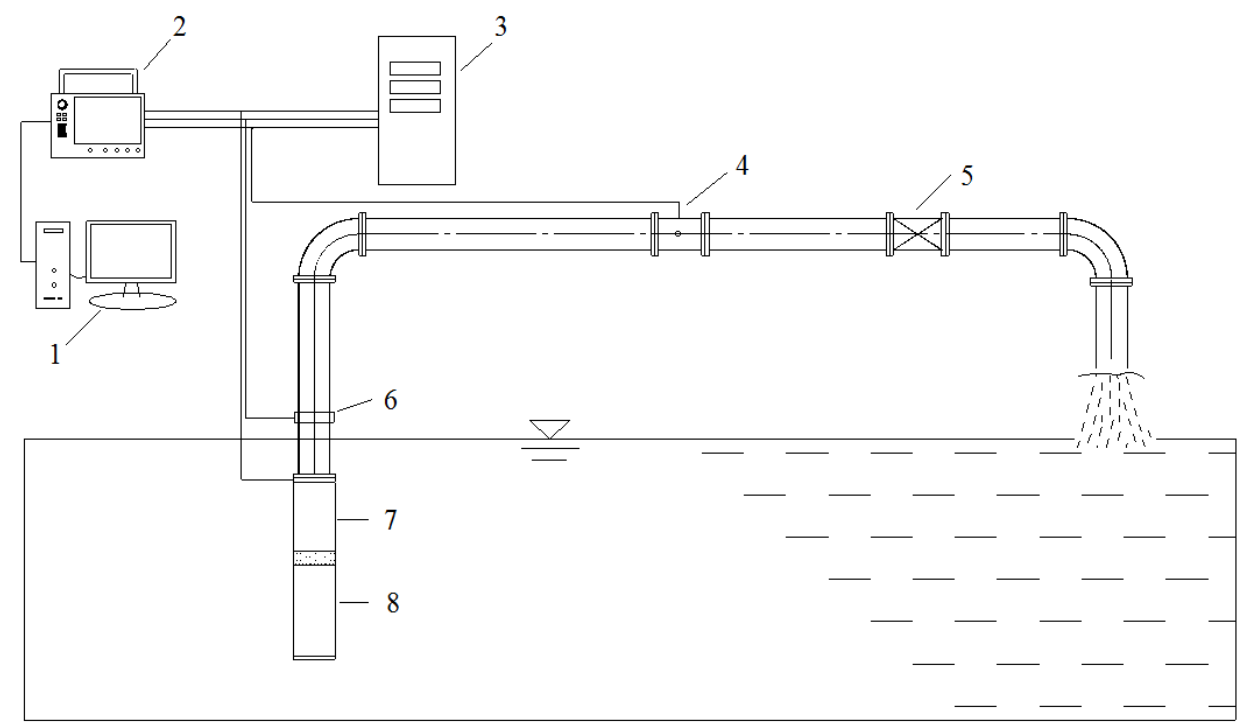

1. Computer 2. Data acquisition instrument 3. Power distribution cabinet and frequency converter module 4. Turbine flowmeter 5. Regulating valve 6. Pressure sensor 7. Pump body 8 . Motor

Figure 3: Schematic of test rig.

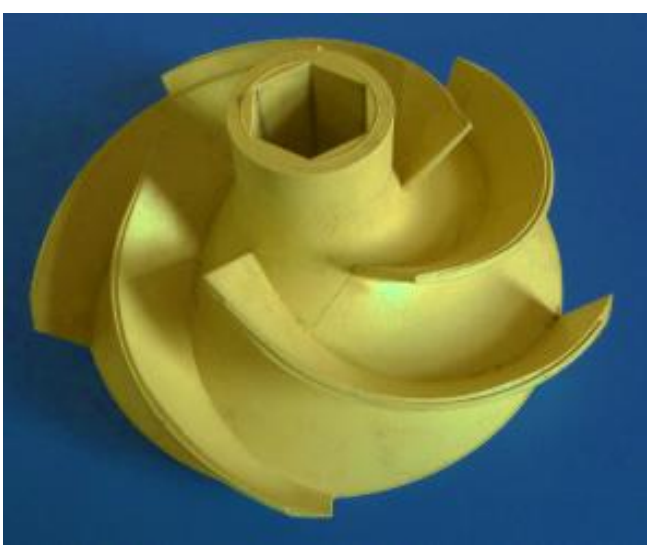

a) Impeller

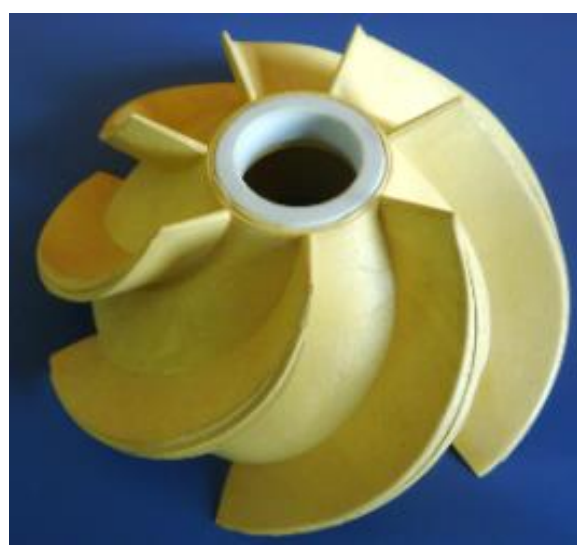

b) Diffuser

Figure 4: Solid model of impeller and diffuser.

Fig. 5 shows the comparison between the experimental and numerical results. The numerical predicted head of the ESP is greater than the test head under full flow conditions. The numerical predicted shaft power is slightly greater than the test power. However, the numerical predicted efficiency is extremely close to the test results. The variation trend of numerical predicted performance under full flow conditions is highly consistent with the variation trend of experimental results. Therefore, the numerical calculation results in this article have certain accuracy. 


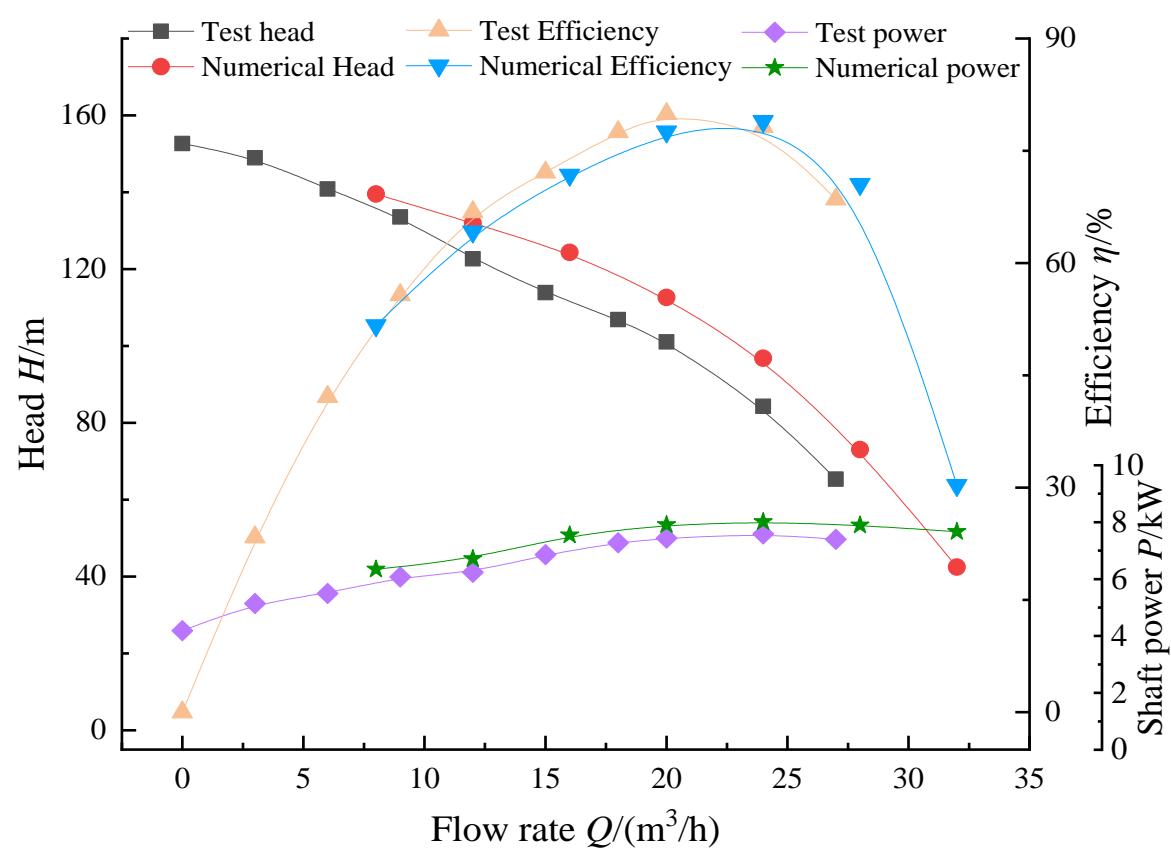

Figure 5: Comparisons between test and numerical results.

\section{RESULTS ANALYSIS AND DISCUSSION}

Fig. 6 shows the three methods of speed variation set in the transient numerical calculations. The speed change of scheme 1 is in linear uniform acceleration. The speed change of scheme 2 is in quadratic curve change with a positive quadratic coefficient. The speed change of scheme 3 is in quadratic curve change with a negative quadratic coefficient. In the three variation methods, the time of speed variation process is $1 \mathrm{~s}$. After the end of speed variation process, the ESP models in all three schemes keep running with $7000 \mathrm{r} / \mathrm{min}$ until the end of the numerical calculation. The setting of speed variation in all three schemes is realized on CEL language in CFX as follows:

During the speed variation of ESP, the flow rate varies with the speed. Thus, the variation of flow rate needs to be controlled by the CEL expression when setting the boundary conditions. In this article, the variation law of flow rate is obtained in accordance with the relationship between flow rate and rotational speed by the similar law.

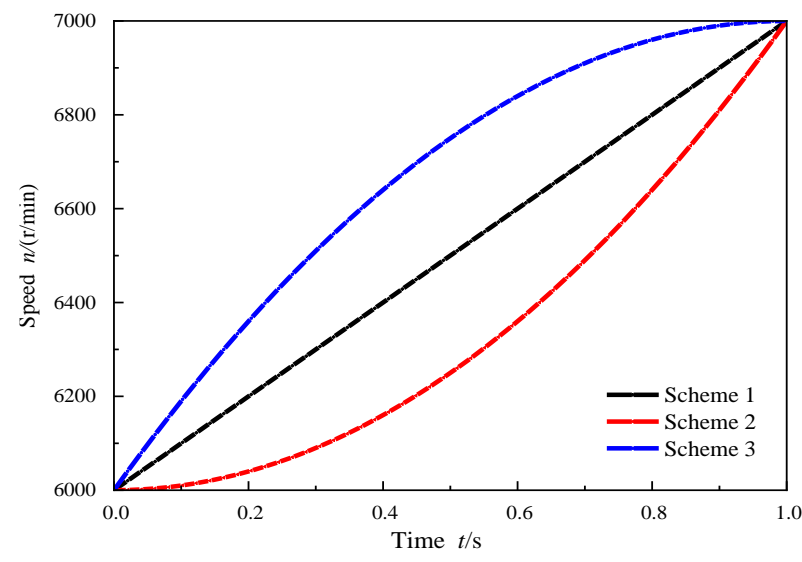

Figure 6: Variation rule of rotational speed.

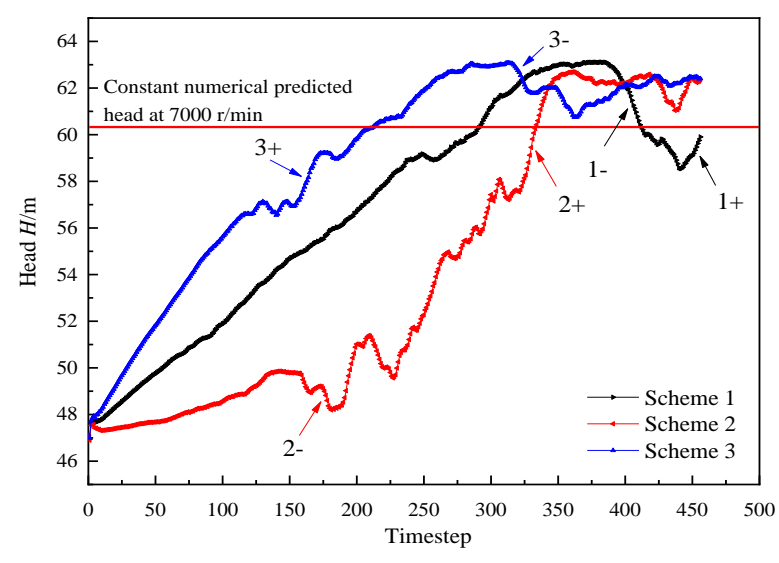

Figure 7: Head change curve.

The transient predicted values of ESP's head with the three rotational speed variations are shown in Fig. 7. A strong similarity is found between the curve variation pattern of the ESP's 
head with the three schemes and the variation pattern of rotational speed curve in Fig. 6. During the speed variation process, the speed of scheme 3 is the highest, the speed of scheme 1 is ranks second, and the speed of scheme 2 is the lowest at the same moment. This finding is the same as the head variation pattern of each option during the speed variation process. The head prediction curve of scheme 1 is similar to its corresponding speed variation curve, with a small fluctuation only in the second half of the speed variation process. The head prediction curve of scheme 2 and its corresponding speed change curve have the same change law in the first third section of the variable speed process. In the middle and second half of the speed change process, the head prediction value has a large fluctuation, and the fluctuation of head is irregular, indicating that the speed change process of the ESP is a nonconstant process with strong transient characteristics. The head prediction curve in scheme 3 and its corresponding speed variation curve have the same variation pattern throughout the speed variation process. However, it shows head fluctuation in the middle of the speed variation process. Comparing the predicted heads under three different schemes, the head in scheme 3 is the first to reach the head of the constant numerical predicted head at $7000 \mathrm{r} / \mathrm{min}$, followed by scheme 1 and scheme 2. The maximum head that can be achieved during the speed variation of the three schemes exceeds the head of the model during the stable operation at $7000 \mathrm{r} / \mathrm{min}$. This condition is because during the variable speed operation of the ESP, the pump head consists of the basic head (the head of the pump stable operation at that speed) and the inertia head (generated by the inertia force during the acceleration of the pump). The maximum head values of the three schemes are the same. However, scheme 3 takes the shortest time to reach the maximum head, and scheme 2 takes the longest time to reach the maximum head.

The head change rate reflects the intensive change degree of head at different moments and is defined as:

$$
\text { Head change rate } H \mathrm{c}=\frac{\Delta H}{\Delta t}(\Delta t \rightarrow 0)
$$

After obtaining the variation of the pump head at different rotational speeds, the change rate of the head at different moments can be calculated and plotted as a curve by using the differentiate function in the Mathematics module of Origin 9.0 software. The variation of the head change rate for the three schemes is shown in Fig. 8.

Comparing the change rate of head with three different schemes, the fluctuation of the change rate of head with time is most obvious for scheme 2 . The positive and negative peaks of the change rate of the head appear at points $2+$ and $2-$ in Fig. 8, corresponding to the sudden rise and fall of the head in Fig. 7, respectively. Within the ESP, the sudden drop and rise of the head may lead to rapid pressure changes in the pump system pipeline, thereby resulting in system vibration and inducing noise. Compared with scheme 2 , the maximum and minimum values of the head variation rate curves of schemes 1 and 3 are distributed between \pm 0.2 , and their head variation is more stable. The sudden rise and fall of the head in scheme 3 correspond to points $3+$ and $3-$ in Fig. 8, which occur in the middle and end of the acceleration process, respectively. The sudden rise and fall of the head in scheme 1 occur at the end of the acceleration process.

Considering the time that the ESP's head reaches the required value and the change rate of head in the process of speed variation, schemes 1 and 3 can be adopted for the variable speed process of ESP. The advantage of scheme 1 is that the head change is smooth during the speed change. However, it has a slightly larger head fluctuation in the late period, and its time to reach the required head is longer than that of scheme 3 . The advantage of scheme 3 is that in the early period of the speed change process, the head is raised fast and can meet the head requirement fast. However, its head fluctuates in the middle part of the change process. 


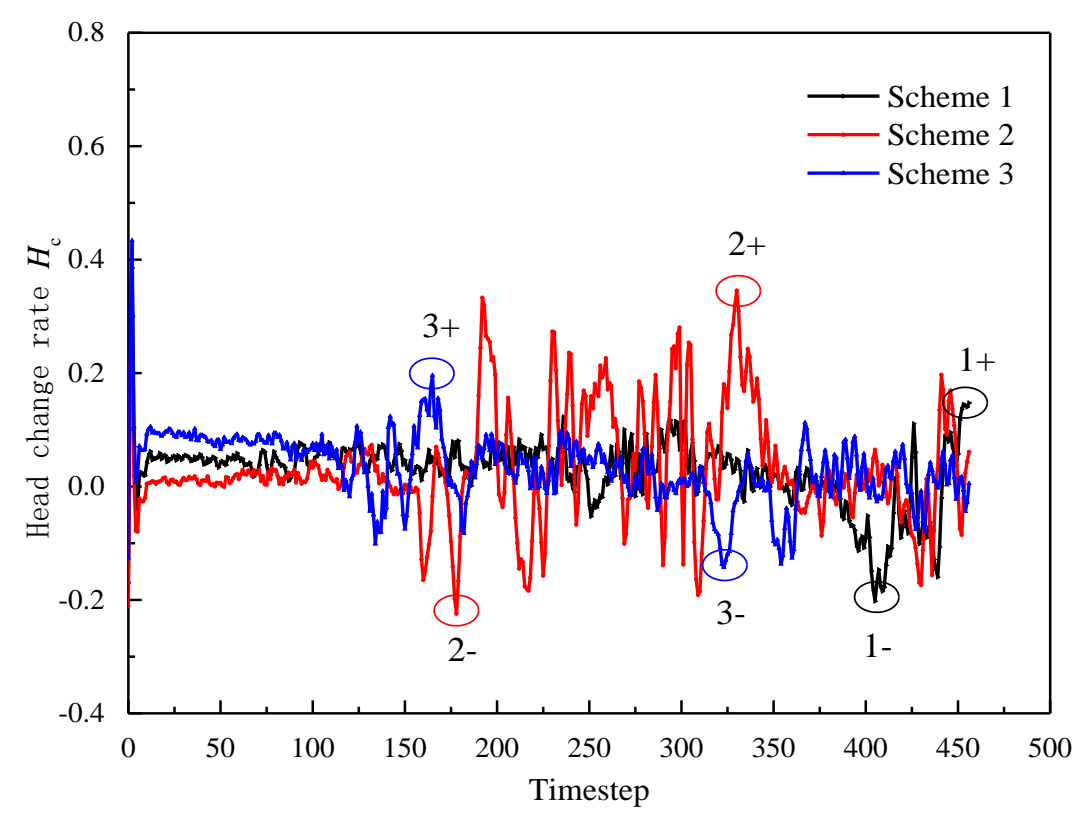

Figure 8: Change rate of head curve.

\section{CONCLUSION}

The influence mechanism of different variable speed modes on the performance of ESP was investigated to improve the variable speed stability of ESP. Three different schemes of speed variation were designed to analyse the change in head fluctuation of ESP under three different speed variation schemes. The performance verification test of ESP was conducted. The main conclusions obtained are as follows.

1) The speed change mode of ESP plays a key role in the head fluctuation of ESP within variable speed process. Therefore, the head lift speed and the head fluctuation rate within the variable speed process must be considered.

2) In the variable speed process, the head variation curves obtained from different speed variation schemes have great similarity with the corresponding speed variation curves, and the overall variation pattern between the two is the same.

3) The head stability is high in the uniform acceleration process and the quadratic acceleration process with negative quadratic coefficients. The head volatility is high in the quadratic acceleration process with positive quadratic coefficients. Among the three processes, the quadratic acceleration process with negative quadratic coefficient has the shortest time to reach the required head.

Numerical calculations were combined with experimental verification in this study. The numerical model for the performance analysis of ESP was presented. The speed variation process, which is an important factor affecting the operational stability of ESP, was investigated. The results serve as guide for the improvement of ESPs' variable speed stability. Only three different variable speed schemes were designed to analyse the influence of variable speed mode on the ESP's operating stability. Thus, many other variable speed modes can be adopted in the follow-up studies.

\section{ACKNOWLEDGEMENT}

The study was supported by the National Natural Science Foundation of China (No. 52009013), Changzhou Sci\&Tech Program (Grant No. CJ20190048) and the Jiangsu Province Graduate Practice Innovation Project (No. SJCX18_0743). 


\section{REFERENCES}

[1] Romero, O. J.; Hupp, A. (2014). Subsea electrical submersible pump significance in petroleum offshore production, Journal of Energy Resources Technology, Vol. 136, No. 1, Paper 012902, 8 pages, doi:10.1115/1.4025258

[2] Bianchini, A.; Rossi, J.; Antipodi, L. (2018). A procedure for condition-based maintenance and diagnostics of submersible well pumps through vibration monitoring, International Journal of System Assurance Engineering and Management, Vol. 9, No. 5, 999-1013, doi:10.1007/s13198$\underline{018-0711-3}$

[3] Zhou, L.; Wang, W.; Hang, J.; Shi, W.; Yan, H.; Zhu, Y. (2020). Numerical investigation of a highspeed electrical submersible pump with different end clearances, Water, Vol. 12, No. 4, Paper 1116, 16 pages, doi: $10.3390 /$ w12041116

[4] Zhu, J.; Guo, X.; Liang, F.; Zhang, H.-Q. (2017). Experimental study and mechanistic modeling of pressure surging in electrical submersible pump, Journal of Natural Gas Science and Engineering, Vol. 45, 625-636, doi:10.1016/j.jngse.2017.06.027

[5] Murillo-Verduzco, I.; Herrera-Sarellano, M.; Figueroa-Diaz, R. A.; Fimbres-Weihs, G. A.; CastroQuintero, R. A. (2020). Design and manufacturing of a pulsatile valve for generating variable flows in reverse osmosis desalination modules, DYNA, Vol. 95, No. 5, 509-513, doi:10.6036/9486

[6] Patil, A.; Kasprzyk, M.; Delgado, A.; Morrison, G. (2020). Effect of leakage flow path wear on axial thrust in downhole electrical submersible pump unit, Journal of Fluids Engineering, Vol. 142, No. 5, Paper 051202, 11 pages, doi:10.1115/1.4045571

[7] Mohammadzaheri, M.; Tafreshi, R.; Khan, Z.; Ghodsi, M.; Franchek, M.; Grigoriadis, K. (2020). Modelling of petroleum multiphase flow in electrical submersible pumps with shallow artificial neural networks, Ships and Offshore Structures, Vol. 15, No. 2, 174-183, doi:10.1080/ $\underline{17445302.2019 .1605959}$

[8] Abadie, L. M.; Goicoechea Larracoechea, N. (2019). Review and analysis of energy storage systems by hydro-pumping to support a mix of electricity generation with a high percentage of renewables, DYNA, Vol. 94, No. 6, 669-675, doi:10.6036/9182

[9] Worth, D.; Al-Safran, E.; Choudhuri, A.; Al-Jasmi, A. (2019). Assessment of artificial lift methods for a heavy oil field in Kuwait, Journal of Petroleum Science and Engineering, Vol. 180, 835-843, doi:10.1016/j.petrol.2019.06.012

[10] Mrzljak, V. (2018). Low power steam turbine energy efficiency and losses during the developed power variation, Technical Journal, Vol. 12, No. 3, 174-180, doi:10.31803/tg-20180201002943

[11] Hu, Q.; Yang, Y.; Shi, W. D. (2020). Cavitation simulation of centrifugal pump with different inlet attack angles, International Journal of Simulation Modelling, Vol. 19, No. 2, 279-290, doi:10.2507/IJSIMM19-2-516

[12] Šumiga, I.; Srpak, D.; Kondić, Ž. (2018). Application of thermoelectric modules as renewable energy sources, Technical Journal, Vol. 12, No. 3, 151-158, doi:10.31803/tg-20180301181527

[13] Yang, Y.; Zhou, L.; Hang, J.; Du, D.; Shi, W.; He, Z. (2021). Energy characteristics and optimal design of diffuser meridian in an electrical submersible pump, Renewable Energy, Vol. 167, 718727, doi:10.1016/j.renene.2020.11.143

[14] Zhou, F.-M.; Wang, X.-F. (2016). Effects of staggered blades on the hydraulic characteristics of a 1400-MW canned nuclear coolant pump, Advances in Mechanical Engineering, Vol. 8, No. 8, Paper 1687814016657944, 21 pages, doi:10.1177/1687814016657944

[15] Yang, Y.; Zhou, L.; Shi, W.; He, Z.; Han, Y.; Xiao, Y. (2021). Interstage difference of pressure pulsation in a three-stage electrical submersible pump, Journal of Petroleum Science and Engineering, Vol. 196, Paper 107653, 13 pages, doi:10.1016/j.petrol.2020.107653

[16] Zhu, J.; Banjar, H.; Xia, Z.; Zhang, H.-Q. (2016). CFD simulation and experimental study of oil viscosity effect on multi-stage electrical submersible pump (ESP) performance, Journal of Petroleum Science \& Engineering, Vol. 146, 735-745, doi:10.1016/j.petrol.2016.07.033

[17] Hydren, J. R.; Kithas, A. C.; Park, S. H.; Wever-Pinzon, O.; Selzman, C. H.; Perry, W.; Vargas, C. A. S.; Stehlik, J.; Drakos, S. G.; Richardson, R. S. (2020). Targeting peripheral vascular pulsatility in heart failure patients with continuous-flow left ventricular assist devices: the impact of pump speed, ASAIO Journal, Vol. 66, No. 3, 291-299, doi:10.1097/mat.0000000000001001 
[18] Schmidt, T.; Reiss, N.; Deniz, E.; Feldmann, C.; Hein, A.; Hoffmann, J.-D.; Robesaat, J. I.; Schmitto, J. D.; Willemsen, D.; Müller-von Aschwege, F. (2017). Adaptive pump speed algorithms to improve exercise capacity in patients supported with a left-ventricular assist device, Studies in Health Technology and Informatics, Vol. 236, 235-240, doi:10.3233/978-1-61499-759-7-235

[19] Liao, S.; Wu, E. L.; Neidlin, M.; Li, Z.; Simpson, B.; Gregory, S. D. (2018). The influence of rotary blood pump speed modulation on the risk of intraventricular thrombosis, Artificial Organs, Vol. 42, No. 10, 943-953, doi:10.1111/aor.13330

[20] Ge, L.; Quan, L.; Zhang, X.; Zhao, B.; Yang, J. (2017). Efficiency improvement and evaluation of electric hydraulic excavator with speed and displacement variable pump, Energy Conversion and Management, Vol. 150, 62-71, doi:10.1016/j.enconman.2017.08.010

[21] Page, P. R.; Abu-Mahfouz, A. M.; Mothetha, M. L. (2017). Pressure management of water distribution systems via the remote real-time control of variable speed pumps, Journal of Water Resources Planning and Management, Vol. 143, No. 8, Paper 04017045, 6 pages, doi:10.1061/(asce)wr.1943-5452.0000807

[22] Nag, S.; Lee, K. Y. (2018). DFIM-based variable speed operation of pump-turbines for efficiency improvement, IFAC-PapersOnLine, Vol. 51, No. 28, 708-713, doi:10.1016/j.ifacol.2018.11.788

[23] Morabito, A.; de Oliveira e Silva, G.; Hendrick, P. (2019). Deriaz pump-turbine for pumped hydro energy storage and micro applications, Journal of Energy Storage, Vol. 24, Paper 100788, 16 pages, doi:10.1016/j.est.2019.100788

[24] Bai, Y.; Kong, F.; Zhao, F.; Wang, J.; Xia, B.; Hu, Q. (2018). Experiments and numerical simulation of performances and internal flow for high-speed rescue pump with variable speeds, Mathematical Problems in Engineering, Vol. 2018, Paper 9168694, 18 pages, doi:10.1155/ 2018/9168694

[25] Al-Obaidi, A. R. (2019). Investigation of effect of pump rotational speed on performance and detection of cavitation within a centrifugal pump using vibration analysis, Heliyon, Vol. 5, No. 6, Paper E01910, 19 pages, doi:10.1016/j.heliyon.2019.e01910

[26] Li, W.; Ji, L.; Ma, L.; Yang, Y.; Zhou, L.; Agarwal, R. (2020). Numerical and experimental study of variable speed automobile engine cooling water pump, Science Progress, Vol. 103, No. 2, Paper 003685042092522, 27 pages, doi:10.1177/0036850420925227

[27] Wang, Z.; Qian, Z.; Lu, J.; Wu, P. (2019). Effects of flow rate and rotational speed on pressure fluctuations in a double-suction centrifugal pump, Energy, Vol. 170, 212-227, doi:10.1016/ j.energy.2018.12.112

[28] Yang, Y.; Zhou, L.; Shi, W.; Wang, C.; Li, W.; Agarwal, R. (2019). Effect of rotating speed on performance of electrical submersible pump, Proceedings of the $8^{\text {th }}$ ASME-JSME-KSME Joint Fluids Engineering Conference, Vol. 3B, Paper AJKFluids2019-5093, 8 pages, doi:10.1115/ ajkfluids2019-5093

[29] Han, Y.; Tan, L. (2020). Influence of rotating speed on tip leakage vortex in a mixed flow pump as turbine at pump mode, Renewable Energy, Vol. 162, 144-150, doi:10.1016/j.renene.2020.08.033

[30] He, X.; Zhang, Y.; Wang, C.; Zhang, C.; Cheng, L.; Chen, K.; Hu, B. (2020). Influence of critical wall roughness on the performance of double-channel sewage pump, Energies, Vol. 13, No. 2, Paper 464, 20 pages, doi:10.3390/en13020464

[31] Wang, H.; Long, B.; Yang, Y.; Xiao, Y.; Wang, C. (2020). Modelling the influence of inlet angle change on the performance of submersible well pumps, International Journal of Simulation Modelling, Vol. 19, No. 1, 100-111, doi:10.2507/IJSIMM19-1-506

[32] Larsson, I. A. S.; Lindmark, E. M.; Lundström, T. S.; Nathan, G. J. (2011). Secondary flow in semi-circular ducts, Journal of Fluids Engineering, Vol. 133, No. 10, Paper 101206, 8 pages, doi: $10.1115 / 1.4004991$ 